\title{
Estudo da audição de ritmistas de uma escola de samba de São Paulo
}

\section{Study of the hearing of percussionists of a samba school from São Paulo}

\author{
Viviane Maria Monteiro ${ }^{1}$, Alessandra Giannella Samelli²
}

\begin{abstract}
RESUMO
Objetivo: Investigar a presença de possíveis perdas auditivas em ritmistas de uma escola de samba de São Paulo. Métodos: O presente estudo foi prospectivo. Participaram dez ritmistas da bateria da escola de samba, de faixa etária entre 20 e 31 anos de idade. Foram realizadas medição dos níveis de pressão sonora dentro da quadra e avaliações audiológicas dos participantes. Resultados: A média dos níveis de pressão sonora na quadra da escola de samba durante os ensaios foi de 111,42 dBA. Seis indivíduos apresentaram limiares auditivos dentro dos limites da normalidade em ambas as orelhas (sendo que as frequências altas apresentaram-se elevadas quando comparadas às frequências médias e baixas); três apresentaram perdas auditivas leves unilaterais; um apresentou perda auditiva neurossensorial leve em ambas as orelhas. Conclusão: Quatro ritmistas da escola de samba apresentaram algum grau de perda auditiva.
\end{abstract}

Descritores: Perda auditiva; Perda auditiva provocada por ruído; Ruído ocupacional; Música

\section{INTRODUÇÃO}

Em virtude das várias horas diárias de ensaios, bem como das frequentes apresentações, os músicos, independente do estilo, em consequência da exposição sistemática a níveis de pressão sonora elevados causada pela música, podem apresentar alterações auditivas que podem prejudicar tanto sua vida profissional como pessoal ${ }^{(1-3)}$.

Segundo a Portaria $19^{(4)}$, que altera a Norma Regulamentadora (NR) 7, do Capítulo V da Consolidação das Leis do Trabalho (Da Segurança e da Medicina do Trabalho), "entende-se por perda auditiva induzida por níveis de pressão sonora elevados as alterações dos limiares auditivos, do tipo sensorioneural, decorrente da exposição ocupacional sistemática a níveis de pressão sonora elevados. Tem como características principais a irreversibilidade e a progressão gradual com o tempo de exposição ao risco. A sua história natural mostra, inicialmente, o acometimento dos limiares auditivos em uma ou mais frequências da faixa de 3000 a $6000 \mathrm{~Hz}$. As frequências mais altas

Trabalho realizado no Curso de Fonoaudiologia da Universidade Guarulhos - UnG - Guarulhos (SP), Brasil.

(1) Fonoaudióloga clínica - Guarulhos (SP), Brasil.

(2) Doutora, Professora do Curso de Fonoaudiologia do Departamento de Fisioterapia, Fonoaudiologia e Terapia Ocupacional da Universidade de São Paulo - USP - São Paulo (SP), Brasil.

Endereço para correspondência: Alessandra Gianella Samelli. Centro de Docência e Pesquisa em Fisioterapia, Fonoaudiologia e Terapia Ocupacional.

R. Cipotânea, 51, Cidade Universitária, São Paulo (SP), Brasil, CEP: 05360-

160. E-mail: alesamelli@usp.br

Recebido em: 5/1/2009; Aceito em: 27/5/2009 e mais baixas poderão levar mais tempo para serem afetadas".

Além da perda auditiva, a exposição de longo termo ao ruído traz outros efeitos para a saúde como: mudanças na respiração, no sono, na função cardiovascular, doenças gastrointestinais, prejuízo para o sistema imunológico, irritação, cansaço, ansiedade, entre outros prejuízos para a saúde física e mental ${ }^{(5-9)}$.

A música, embora não seja classificada como ruído, quando executada em níveis de pressão sonora elevados, pode lesar o sistema auditivo de forma progressiva e permanente ${ }^{(10)}$. Embora o ruído frequentemente referido como lesivo seja o ocupacional, qualquer fonte de som com níveis de pressão sonora superiores a $85 \mathrm{~dB}(\mathrm{~A})$ pode ser prejudicial à audição e ao indivíduo, dependendo do tempo diário e da periodicidade desta exposição. Desta forma, dependendo destas variáveis (intensidade, tempo e periodicidade), o músico pode desenvolver perda auditiva, havendo também interferência da variabilidade individual na susceptibilidade à lesão auditiva ${ }^{(1,11-12)}$.

Segundo revisão da literatura sobre o assunto ${ }^{(13)}$, os músicos costumam estar expostos a níveis de pressão sonora variados, conforme o estilo de música, o local de apresentação, o(s) instrumento(s) utilizado(s), a posição do músico dentro da banda ou orquestra, dentre outros. Desta forma, os níveis de pressão sonora variam desde aproximadamente $81,4 \mathrm{~dB}(\mathrm{~A})$ em orquestras sinfônicas ${ }^{(1)}$ até $119 \mathrm{~dB}(\mathrm{~A})$ em grupos de Maracatu $^{(14)}$.

A NR $15^{(15)}$, que trata das Atividades e Operações Insalubres, recomenda que não haja exposição acima de $85 \mathrm{~dB}(\mathrm{~A})$ por mais de oito horas de ruído contínuo ou intermitente. Para 
cada $5 \mathrm{~dB}(\mathrm{~A})$ acrescido no nível de ruído, o tempo de exposição deve cair pela metade. Sendo assim, para uma exposição a níveis de $115 \mathrm{~dB}(\mathrm{~A})$, o máximo de exposição permitida seria sete minutos. Com base nestas recomendações, fica notório que os músicos, de forma geral, estão expostos a níveis de pressão sonora potencialmente lesivos à audição, pois excedem o recomendado para a saúde auditiva.

Vários estudos descreveram diversas alterações auditivas em músicos ou profissionais que trabalham com música amplificada: prejuízo da função coclear observado por meio de emissões otoacústicas ${ }^{(1-2,16,17)}$; perda auditiva neurossensorial nas frequências altas verificada por meio de audiometria tonal ${ }^{(1-3,11-12,14,18)}$; e queixas auditivas (principalmente zumbido) ${ }^{1-2,12,14)}$.

Por isso, o objetivo deste estudo foi investigar a presença de possíveis perdas auditivas em ritmistas de uma escola de samba de São Paulo.

\section{MÉTODOS}

O estudo foi iniciado após a aprovação do projeto de pesquisa pelo Comitê de Ética em Pesquisa da Universidade de Guarulhos (Parecer $n^{\circ}$ 116/2006).

Participaram deste estudo dez ritmistas da bateria da escola de samba, sendo cinco do gênero masculino e cinco do gênero feminino, de faixa etária entre 20 e 31 anos de idade (média de 27,5 anos), com tempo médio nesta função de 4,7 anos (Quadro 1).

Os critérios de inclusão do estudo foram: idade entre 18 e 35 anos, tempo de exposição ao ruído acima de três anos, não possuir antecedentes de exposição ocupacional além da música, bem como queixas auditivas ou realização de cirurgias otológicas, além de ausência de doenças sistêmicas conhecidas ou de perdas auditivas condutivas.

Inicialmente, foi obtido o consentimento livre e esclarecido dos participantes desta pesquisa. A primeira parte do estudo envolveu a convocação dos participantes, que foi feita mediante convite de músicos que tocavam diferentes instrumentos. Este convite foi realizado de forma aleatória, por meio de uma lista fornecida pela escola de samba. Posteriormente, foi feita também a medição do nível de pressão sonora na quadra, durante os ensaios. A segunda parte referiu-se à avaliação audiológica dos músicos.

Para a medição do ruído na quadra da escola de samba, foi utilizado o medidor de nível de pressão sonora Realistic ${ }^{\circledR}$ (CAT 332055), na escala A. A medição foi feita durante um ensaio geral da escola de samba. $\mathrm{O}$ medidor foi posicionado na altura das orelhas dos integrantes da bateria com distância de aproximadamente meio metro. Cada medição foi composta por doze amostras de níveis de pressão sonora, feitas durante um minuto, a cada cinco segundos ${ }^{(19)}$.

$\mathrm{Na}$ segunda etapa deste estudo, os participantes responderam a uma anamnese elaborada especificamente para este estudo, contendo questões sobre saúde ocupacional e hábitos de vida, histórico médico, bem como questões sobre a atividade na escola de samba. Em seguida, foram submetidos à avaliação audiológica. Os procedimentos foram realizados com os participantes em repouso acústico de, no mínimo, 16 horas. Primeiramente foi realizada a meatoscopia, para descartar quaisquer alterações de orelha externa e/ou média, que pudessem influenciar na coleta dos dados; o otoscópio utilizado foi o Heine ${ }^{\circledR}$ Mini 2000. Depois, foram obtidos os limiares tonais por via aérea (frequências de 250 a $8000 \mathrm{~Hz}$ ) e por via óssea (frequências de $500 \mathrm{a} 4000 \mathrm{~Hz}$ ), quando necessário, bem como o limiar de recepção de fala e o índice percentual de reconhecimento de fala, com a utilização do audiômetro da marca Interacoustics ${ }^{\circledR}$, modelo AC 33 . Tanto a audiometria tonal quanto a audiometria vocal foram realizadas em cabina acústica. Foi realizada também a imitanciometria (timpanometria e a pesquisa dos reflexos acústicos ipsi e contralaterais), com o aparelho da marca Interacoustics $\AA$, modelo AZ 7. Todos os resultados foram registrados em folha de exame específica.

Como critério de classificação dos limiares auditivos, foi utilizada a Portaria 19(4): "São considerados sugestivos de perda auditiva induzida por níveis de pressão sonora elevados os casos cujos audiogramas, nas frequências de 3000 e/ ou 4000 e/ou $6000 \mathrm{~Hz}$, apresentam limiares auditivos acima de $25 \mathrm{~dB}(\mathrm{NA})$ e mais elevados do que nas outras frequências testadas, estando estas comprometidas ou não, tanto no teste da via aérea quanto da via óssea, em um ou em ambos os lados."

Para análise estatística, foi aplicado o teste ANOVA (Análise da Variância), com nível de significância de 5\% ou p <0,05.

Quadro 1. Gênero, idade, instrumento tocado e tempo de exposição à música por ritmista

\begin{tabular}{|lcccc|}
\hline $\mathrm{N}^{\circ}$ & Gênero & Idade (em anos) & Instrumentos que tocam & Tempo de exposição (em anos) \\
\hline 1 & Feminino & 31 & Caixa & 7 \\
2 & Feminino & 20 & Chocalho & 5 \\
3 & Masculino & 28 & Cuíca & 3 \\
4 & Masculino & 20 & Caixa & 3 \\
5 & Masculino & 30 & Surdo de 1 & 6 \\
6 & Masculino & 26 & Tamborim & 4 \\
7 & Masculino & 28 & Surdo de 2 & 3 \\
8 & Feminino & 23 & Surdo de 3 & 5 \\
9 & Feminino & Tamborim & 4 \\
\hline
\end{tabular}




\section{RESULTADOS}

\section{Níveis de pressão sonora durante ensaio da escola de samba}

Conforme se observa na Tabela 1, a média geral de nível de pressão sonora na quadra da escola de samba durante o ensaio foi de 111,42 dBA.

Tabela 1. Medições dos níveis de pressão sonora dentro da quadra da escola de samba

\begin{tabular}{lccc}
\hline Amostras & $\begin{array}{c}1^{\mathrm{a}} \text { medição } \\
(\mathrm{dBA})\end{array}$ & $\begin{array}{c}2^{\mathrm{a}} \text { medição } \\
(\mathrm{dBA})\end{array}$ & $\begin{array}{c}3^{\mathrm{a}} \text { medição } \\
(\mathrm{dBA})\end{array}$ \\
\hline 1 & 111 & 112 & 112 \\
2 & 111 & 113 & 112 \\
3 & 111 & 112 & 112 \\
4 & 111 & 112 & 111 \\
5 & 111 & 113 & 112 \\
6 & 111 & 112 & 111 \\
7 & 111 & 111 & 112 \\
8 & 111 & 111 & 112 \\
9 & 112 & 112 & 111 \\
10 & 110 & 112 & 110 \\
11 & 110 & 111 & 111 \\
12 & 109 & 112 & 113 \\
\hline Média por medição & 110,75 & 111,92 & 111,58 \\
\hline Média geral & & 111,42 & \\
\hline
\end{tabular}

Legenda: $\mathrm{dBA}$ = decibéis nível de audição

\section{Dados da anamnese}

De acordo com as respostas obtidas no questionário, no que se refere ao uso de protetor auricular, $100 \%$ dos participantes não utilizam este equipamento de proteção individual durante os ensaios da escola de samba; $90 \%$ percebem zumbido e/ou diminuição da audição após os ensaios da escola de samba.

\section{Avaliação audiológica}

De acordo com os limiares audiométricos dos participantes, pôde-se verificar que seis indivíduos apresentaram limiares auditivos dentro dos limites da normalidade em ambas as orelhas (60\%); dois apresentaram perdas auditivas leves (com característica neurossensorial) na frequência de $6000 \mathrm{~Hz}$ em orelha esquerda e um em orelha direita (30\%); e 1 (10\%), apresentou perda auditiva neurossensorial leve nas frequências de 4000 a $8000 \mathrm{~Hz}$ em ambas as orelhas (Tabela 2). Resumindo, 40\% dos participantes apresentaram algum grau de perda auditiva. A análise estatística mostrou que não houve diferença estatisticamente significante entre os limiares auditivos das duas orelhas.

É importante ressaltar a configuração dos audiogramas, onde as frequências altas apresentam-se elevadas quando comparadas às frequências médias e baixas, mesmo quando os limiares auditivos estavam dentro dos limites de normalidade.

\section{DISCUSSÃO}

O presente estudo evidenciou que os níveis de pressão sonora presentes na quadra da escola de samba durante os ensaios são extremamente elevados, ou seja, com média acima de $111 \mathrm{dBA}$, o que é potencialmente lesivo para a audição. De acordo com a NR $15^{(15)}$, uma pessoa pode ficar exposta a estes níveis de pressão sonora por aproximadamente 12 minutos. Durante um ensaio, o tempo de exposição é muito superior a este limite, podendo chegar a cinco horas. A periodicidade dos ensaios é variável, de duas a quatro vezes por semana, tornando-se quase diária nos meses que antecedem o carnaval.

Tabela 2. Limiares auditivos dos participantes, médias, desvio-padrão e p-valor por frequência nas orelhas direita e esquerda

\begin{tabular}{|c|c|c|c|c|c|c|c|c|c|c|c|c|c|c|c|c|}
\hline \multirow{3}{*}{$\mathrm{N}$} & \multicolumn{16}{|c|}{ Limiares auditivos tonais por via aérea (dB NA) } \\
\hline & \multicolumn{2}{|c|}{$250 \mathrm{~Hz}$} & \multicolumn{2}{|c|}{$500 \mathrm{~Hz}$} & \multicolumn{2}{|c|}{$1 \mathrm{kHz}$} & \multicolumn{2}{|c|}{$2 \mathrm{kHz}$} & \multicolumn{2}{|c|}{$3 \mathrm{kHz}$} & \multicolumn{2}{|c|}{$4 \mathrm{kHz}$} & \multicolumn{2}{|c|}{$6 \mathrm{kHz}$} & \multicolumn{2}{|c|}{$8 \mathrm{kHz}$} \\
\hline & OD & OE & OD & OE & OD & OE & OD & OE & OD & OE & OD & $\mathrm{OE}$ & OD & OE & OD & OE \\
\hline 1 & 5 & 5 & 0 & 10 & 5 & 5 & 20 & 15 & 15 & 5 & 15 & 0 & 30 & 25 & 15 & 10 \\
\hline 2 & 10 & 15 & 5 & 10 & 5 & 10 & 0 & 0 & 0 & 5 & 0 & 0 & 15 & 0 & 10 & 5 \\
\hline 3 & 5 & 5 & 10 & 5 & 5 & 5 & 5 & 5 & 10 & 15 & 10 & 15 & 10 & 20 & 0 & 0 \\
\hline 4 & 0 & 0 & 0 & 0 & 0 & 0 & 15 & 0 & 10 & 0 & 5 & 0 & 5 & 0 & 5 & 0 \\
\hline 5 & 10 & 5 & 10 & 10 & 5 & 10 & 10 & 5 & 20 & 10 & 25 & 15 & 25 & 30 & 20 & 20 \\
\hline 6 & 5 & 0 & 10 & 5 & 15 & 5 & 10 & 5 & 10 & 10 & 15 & 10 & 15 & 15 & 10 & 10 \\
\hline 7 & 5 & 10 & 10 & 5 & 15 & 10 & 10 & 15 & 10 & 10 & 10 & 20 & 5 & 25 & 10 & 15 \\
\hline 8 & 10 & 10 & 15 & 5 & 10 & 10 & 10 & 15 & 15 & 15 & 15 & 20 & 20 & 30 & 15 & 15 \\
\hline 9 & 0 & 5 & 0 & 0 & 5 & 0 & 10 & 5 & 5 & 5 & 5 & 5 & 0 & 0 & 5 & 0 \\
\hline 10 & 5 & 10 & 10 & 10 & 5 & 10 & 10 & 15 & 15 & 15 & 35 & 20 & 30 & 40 & 25 & 30 \\
\hline Média & 5,5 & 6,5 & 7 & 6 & 7 & 6,5 & 10 & 8 & 11 & 9 & 13,5 & 10,5 & 15,5 & 18,5 & 11,5 & 10,5 \\
\hline DP & 3,68 & 4,74 & 5,37 & 3,94 & 4,83 & 4,11 & 5,27 & 6,32 & 5,67 & 5,16 & 10,28 & 8,64 & 10,65 & 14,34 & 7,47 & 9,84 \\
\hline Valor 0 & \multicolumn{2}{|c|}{0,535} & \multicolumn{2}{|c|}{0,136} & \multicolumn{2}{|c|}{0,583} & \multicolumn{2}{|c|}{0,428} & \multicolumn{2}{|c|}{0,382} & \multicolumn{2}{|c|}{0,468} & \multicolumn{2}{|c|}{0,588} & \multicolumn{2}{|c|}{0,784} \\
\hline
\end{tabular}

Legenda: $O D$ = orelha direita; $O E$ = orelha esquerda; $D P=$ desvio-padrão. Teste ANOVA 
Todos estes fatores, associados à falta de uso de um protetor auricular, como constatado em todos os participantes do estudo, sugerem que estes músicos estão expostos a um risco iminente para a audição, conforme descrito em vários estudos $^{(1,11,13-14,16,20)}$.

Sabe-se que a mudança temporária de limiar é comum após a exposição a níveis de pressão sonora elevados e que melhora gradualmente, nas horas seguintes à exposição. Dentre os sintomas desta mudança temporária de limiar, têm-se a sensação de diminuição da audição e o zumbido(13-14,20-22). Estes dois sintomas foram relatados por $90 \%$ dos indivíduos do presente estudo, sugerindo que a maioria dos participantes sofre mudanças temporárias de audição após os ensaios da escola de samba.

Com o passar do tempo, dependendo do tempo de exposição e dos níveis de pressão sonora, esta mudança de limiar pode deixar de ser reversível e se tornar uma perda auditiva permanente, configurando uma Perda Auditiva Induzida por Níveis de Pressão Sonora Elevados (PAINPSE) ou, no caso dos músicos, Perda Auditiva Induzida pela Música (PAIM) ${ }^{(13,20)}$.

Dentre os participantes do estudo, $40 \%$ apresentaram algum grau de perda auditiva neurossensorial, com características sugestivas de perda auditiva induzida por níveis de pressão sonora elevados, já que nestes casos, as "frequências de 3000 e/ou 4000 e/ou $6000 \mathrm{~Hz}$, apresentaram limiares auditivos acima de $25 \mathrm{~dB}(\mathrm{NA})$ e mais elevados do que nas outras frequências testadas, estando estas comprometidas ou não, tanto no teste da via aérea quanto da via óssea, em um ou em ambos os lados" ${ }^{\prime(4)}$. Estes achados estão de acordo com outros estudos sobre o mesmo tema, sugerindo que os músicos, independente do estilo, podem desenvolver perdas auditivas neurossensoriais, possivelmente relacionadas com a exposição a níveis de pressão sonoras elevados ${ }^{(11,13-14,20)}$.

Com relação à ocorrência de perdas auditivas unilaterais (três casos), observou-se que, durante os ensaios, a maioria dos ritmistas costuma tocar seus instrumentos de um lado de suas cabeças, ou seja, expondo mais uma orelha do que a outra e talvez, por isso, a ocorrência de algumas perdas auditiva unilaterais. Aspectos como direção do som do próprio instrumento e posição que o músico toca em relação ao grupo já foram anteriormente descritos como sendo fatores para aumento dos níveis de pressão sonora individuais, aumentando a possibilidade de perdas auditivas unilateriais ou assimétricas ${ }^{(3,13)}$.

Foi observado também, mesmo nos casos nos quais os limiares auditivos estavam dentro dos limites de normalidade, que os limiares das frequências altas estavam mais elevados do que nas outras frequências testadas, sugerindo que a exposição à música, executada em níveis de pressão sonora intensos, já tenha produzido algum efeito negativo na audição destes músicos. Estes casos são semelhantes aos estudados por outro trabalho ${ }^{(16)}$, que apresentaram limiares auditivos dentro da normalidade, porém com maior concentração de limiares piores nas frequências altas $(3000,4000$ e $6000 \mathrm{~Hz})$, bem como ausência de emissões otoacústicas transitórias em $61 \%$ dos músicos, o que pode sugerir uma alteração pré-clínica das células ciliadas externas, não verificada na avaliação audiológica convencional, provavelmente em virtude da exposição à música em níveis de pressão sonora elevados ${ }^{(17)}$.

Deve-se ressaltar a faixa etária do grupo em questão, isto é, a média de idade do grupo estudado foi de 27,5 anos, tendo o participante mais velho 31 anos. Sendo assim, pôde-se verificar que este é um grupo jovem, de início de carreira na música (tempo de exposição à música de 4,7 anos, em média). Mesmo com a faixa etária baixa e o pouco tempo de exposição à música, já existem alguns indivíduos com algum grau de comprometimento auditivo.

É evidente que o estudo em questão apresenta um número reduzido de participantes e que estes resultados não podem ser generalizados para os músicos em geral. A presente investigação não tem a pretensão de esgotar o assunto, mas sim chamar a atenção para a importância do tema em questão, num país onde o samba faz parte da cultura. É relevante destacar que, mesmo em um grupo tão pequeno, selecionado ao acaso dentre os participantes da escola de samba, $40 \%$ dos músicos apresentaram alteração auditiva provavelmente causada pela música.

Este fato evidencia a necessidade de se criar programas de conservação auditiva para músicos, uma vez que estes estão

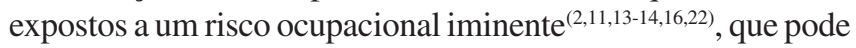
prejudicar a vida profissional e/ou pessoal destes profissionais, causando perdas auditivas bem como outros prejuízos não auditivos como mudanças na respiração, no sono, na função cardiovascular, doenças gastrointestinais, prejuízo para o sistema imunológico, irritação, cansaço, ansiedade, entre outros prejuízos para a saúde física e mental, da mesma forma como ocorre com outras categorias profissionais, também expostas a níveis de pressão sonora elevados ${ }^{(5-9)}$.

Desta forma, aspectos como: medidas de controle de engenharia (revestimento acústico, distância da fonte sonora, diminuição da amplificação, entre outros), medidas de proteção individual (uso de protetores auriculares próprios para músicos, com atenuação uniforme de frequências), treinamento para colocação e higienização dos protetores auriculares, programas educativos, acompanhamento audiológico, entre outros, devem ser instituídos. Existe também a necessidade de criação de leis específicas que regulamentem programas de saúde ocupacional para esta categoria profissional ${ }^{(1-2,11,14,16,20)}$.

\section{CONCLUSÃO}

Com base nos resultados do presente estudo, pôde-se observar que uma porcentagem (40\%) dos ritmistas avaliados de uma escola de samba de São Paulo apresentou perda auditiva. 


\begin{abstract}
Purpose: To investigate possible hearing loss in percussionists of a samba school from São Paulo. Methods: Ten percussionists of a samba school from São Paulo, with ages between 20 and 31 years, participated in this prospective study. Sound pressure levels were measured during a rehearsal and auditory evaluations of the participants were carried out. Results: Mean sound pressure level during rehearsals at the samba school was $111.42 \mathrm{dBA}$. Six subjects showed normal hearing thresholds in both ears (high frequencies more elevated than medium and low frequencies); three showed unilateral hearing loss; one had bilateral sensorineural hearing loss. Conclusion: Four percussionists of the samba school had some degree of hearing loss.
\end{abstract}

Keywords: Hearing loss; Hearing loss, noise-induced; Noise, occupational; Music

\section{REFERÊNCIAS}

1. Nammur FA, Fukuda Y, Onishi ET, Toledo RN. Avaliação auditiva em músicos da Orquestra Sinfônica Municipal de São Paulo. Rev Bras Otorrinolaringol. 1999;65(5):390-5.

2. Maia AA, Gonçalves DU, Menezes LN, Barbosa BMF, Almeida PS, Resende LM. Análise do perfil audiológico dos músicos da Orquestra Sinfônica de Minas Gerais (OSMG). Per Musi. 2007;(15):67-71.

3. Morais D, Benito JI, Almaraz A. Traumatismo acústico en los músicos de música Clásica. Acta Otorrinolaringol Esp. 2007;58(9):401-7.

4. Brasil. Secretaria de Segurança e Saúde no Trabalho. Portaria nº 19, de 9 de abril de 1998. Altera o quadro II - Parâmetros para monitoração da exposição ocupacional a alguns riscos à saúde, da NR 7 - Programa de Controle Médico e Saúde Ocupacional; Inclui o anexo I - Quadro II - Diretrizes e parâmetros mínimos para avaliação e acompanhameto da audição em trabalhadores expostos a níveis de pressão sonora elevados, da NR 7 - Programa de Controle Médico e Saúde Ocupacional [Internet]. Diário Oficial da República Federativa do Brasil, Brasília (DF); 1998 Abr 9 [citado 2008 Ago 28]. Disponível em: www.mte.gov. br/legislacao/Portarias/1998/p_19980409_19.asp.

5. Penney PJ, Earl CE. Occupational noise and effects on blood pressure: exploring the relationship of hypertension and noise exposure in workers. AAOHN J. 2004;52(11):476-80.

6. Kujala T, Shtyrov Y, Winkler I, Saher M, Tervaniemi M, Sallinen M, et al. Long-term exposure to noise impairs cortical sound processing and attention control. Psychophysiology. 2004;41(6):875-81.

7. Brattico E, Kujala T, Tervaniemi M, Alku P, Ambrosi L, Monitillo V. Long-term exposure to occupational noise alters the cortical organization of sound processing. Clin Neurophysiol. 2005;116(1):190203.

8. Hawkins JE, Schacht J. Sketches of otohistory. Part 10: noise-induced hearing loss. Audiol Neurootol. 2005;10(6):305-9.

9. Griest SE, Folmer RL, Martin WH. Effectiveness of "Dangerous Decibels," a school-based hearing loss prevention program. Am J Audiol. 2007;16(2):S165-81.

10. Vansin R, Assencio-Ferreira VJ. Avaliação da alteração do limiar auditivo em jovens frequentadores de casas noturnas. Rev CEFAC. 2002;4(3):219-22.
11. Samelli AG, Schochat E. Perda auditiva induzida por nível de pressão sonora elevado em um grupo de músicos profissionais de rock-and-roll. Acta AWHO. 2000;19(3):136-43.

12. Schmuziger N, Patscheke J, Probst R. Hearing in nonprofessional pop/ rock musicians. Ear Hear. 2006;27(4):321-30.

13. Mendes MH, Morata TC. Exposição profissional à música: uma revisão. Rev Soc Bras Fonoaudiol. 2007;12(1):63-9.

14. Andrade AI, Russo IC, Lima ML, Oliveira LC. Avaliação auditiva em músicos de frevo e maracatu. Rev Bras Otorrinolaringol. 2002;68(5):714-20.

15. Segurança e medicina do trabalho - Normas regulamentadoras [Internet]. São Paulo: Atlas; 1995 [citado 2006 Nov 4]. Disponível em: http://www.mte.gov.br/Empregador/segsau/Legislacao/Normas/Download/ NR_15.pdf. 16. Maia JR, Russo IC. Estudo da audição de músicos de rock and roll. Pró-Fono. 2008;20(1):49-54.

17. Hamdan AL, Abouchacra KS, Zeki Al Hazzouri AG, Zaytoun G. Transient-evoked otoacoustic emissions in a group of professional singers who have normal pure-tone hearing thresholds. Ear Hear. 2008;29(3):360-77.

18. Potier M, Hoquet C, Lloyd R, Nicolas-Puel C, Uziel A, Puel JL. The risks of amplified music for disc-jockeys working in nightclubs. Ear Hear. 2009 Feb 3 [Epub ahead of print].

19. Costa AS, De Martino E, Veloso S, Pires RM. Programa de conservação auditiva em maternidades: resultados parciais referentes às medições do ruído das incubadoras. Rev Unicastelo. 2000;3(3):53-8.

20. Mendes MH, Morata TC, Marques JM. Aceitação de protetores auditivos pelos componentes de banda instrumental e vocal. Rev Bras Otorrinolaringol. 2007;73(3):785-92.

21. Brasil. Ministério da Saúde. Secretaria de Atenção à Saúde. Departamento de Ações Programáticas Estratégicas. Perda auditiva induzida por ruído (Pair). Brasília: Editora do Ministério da Saúde; 2006. Saúde do Trabalhador Protocolos de Complexidade Diferencial, 5 [Série A. Normas e Manuais Técnicos].

22. Pfeiffer M, Rocha RL, Oliveira FR, Frota S. Intercorrência audiológica em músicos após um show de. Rev CEFAC. 2007;9(3):423-9. 